\title{
Administering Anesthesia for Patients with ALS Having a Diaphragmatic Pacemaker Placed: Special Considerations
}

\author{
Molly Kraus, Lopa Misra \\ Mayo Clinic Hospital, Phoenix, USA \\ Email: kraus.molly@mayo.edu
}

Received 13 July 2014; revised 15 August 2014; accepted 5 September 2014

Copyright (C) 2014 by authors and Scientific Research Publishing Inc.

This work is licensed under the Creative Commons Attribution International License (CC BY). http://creativecommons.org/licenses/by/4.0/

\section{(c) (i) Open Access}

\begin{abstract}
Amyotrophic lateral sclerosis (ALS) also known as Lou Gehrig's disease is a relentlessly progressive, fatal disease. Progression of the disease results 3 - 5 years after diagnosis, often from respiratory failure. The diaphragm pacing system (DPS) is a device that stimulates the diaphragm to maximally contract so patients can breathe more effectively. It has been used in patients with neurologic injuries such as spinal cord injury and ALS. From an anesthetic perspective, both the surgery and the patient population present several unique challenges. This case series describes three patients with ALS who had the diaphragmatic pacemaker placed and the anesthetic management during those surgeries.
\end{abstract}

\section{Keywords}

ALS, Amyotrophic Lateral Sclerosis, Diaphragmatic Pacemaker, DPS, Lou Gehrig's Disease

\section{Introduction}

Amyotrophic lateral sclerosis (ALS) also known as Lou Gehrig's disease is a relentlessly progressive, fatal disease involving both upper and lower motor neurons. Motor nerve degeneration occurs in the cerebral cortex, brainstem and spinal cord. Death results typically 3 - 5 years after diagnosis, often from respiratory failure [1]. Respiratory failure can result from inability to cough to clear secretions, carbon dioxide retention, and decrease in ventilatory function. This respiratory compromise can be measured by following pulmonary function tests (PFT). The rate of decline of the forced vital capacity (FVC) has been estimated to be $3 \%-5 \%$ of the predicted values per month [2].

There is little literature available related to ALS and general anesthesia. Historically, it has been considered 
dangerous and thought to be associated with exaggerated ventilatory depression. Even minor surgeries are thought to carry increased risk in patients with ALS. One study reported a thirty-day mortality rate for percutaneous gastrostomy tube placement is as high as $25 \%$ [3].

The diaphragm pacing system (DPS) is a device that stimulates the diaphragm to maximally contract. It is currently being utilized in patients with ALS to improve their ventilatory function and quality of life. This idea of aiding patients' ventilation began in the 1950s, when phrenic nerve stimulation was used to treat patients with polio, with little success. The modern DPS system was initially developed to provide natural negative pressure ventilation in spinal cord-injured patients [4]. All currently available systems involve an external transmitter and an implanted receiver and stimulate the diaphragm to maximally contract. They are highly programmable, delivering stimuli at varying intensities and intervals.

Introduction of DPS before FVC is $<50 \%$ predicted preserves diaphragm strength and prolongs "mechanicalfree ventilation" survival [5]. It also improves quality of life and decreases the rate of decline in FVC in patients with ALS [4]. The diaphragmatic pacemaker is placed laparoscopically, often coupled with the placement of a gastrostomy tube. General anesthesia is necessary for the surgery. During placement, each diaphragm is mapped for a point at which maximal contraction is achieved. Electrodes are then placed, so DPS can assist patient's breathing.

Anesthesiologists face a challenge in this situation: provide adequate relaxant for laparoscopic surgery without the use of neuromuscular blocking drugs. This paper will describe three patients undergoing surgery for the placement of a DPS. It will detail the surgical steps of the surgery. The anesthetic induction, maintenance, and extubation techniques are also outlined. All patients gave informed consent.

\section{Case 1}

A fifty-year-old female with recent diagnosis of ALS with progressive dyspnea on exertion presented for a diaphragmatic pacer insertion and insertion of gastrojejunostomy tube. Prior to surgery, she was started on non-invasive ventilation (NIV) at night. Her recent PFT demonstrated a restrictive pattern with a forced expiratory volume in 1 second (FEV1) of 2.13 (74\% predicted) and FVC of 2.24 (62\% predicted). Maximal inspiratory pressure (MIP) was 36 (41\% predicted).

Induction of anesthesia for this and subsequent cases was achieved with propofol (2 mg/kg) and remifentanil (50 mcg) boluses (Table 1). No neuromuscular blocking drug was used. An endotrachial tube was placed and the patient was ventilated on volume control at $6-8 \mathrm{ml} / \mathrm{kg}$. Anesthesia was maintained with remifentanil infusion (0.1 - $0.2 \mathrm{mcg} / \mathrm{kg} / \mathrm{min}$ ) and sevoflourane (Table 2) [6]. The surgeon inserted ports for laparoscopy and proceeded to map each hemidiaphragm to identify the point of maximal contraction. Once identified, electrodes were implanted and the DPS system tested. A gastrostomy was also created during the surgery. The same surgical steps were followed for the following cases. At the end of the surgery, anesthesia was discontinued and the patient was allowed to breathe spontaneously. The patient was extubated in the operating room when they met extubation criteria: adequate tidal volumes, awake and following commands and strength at or near baseline.

Postoperatively, the patient used the DPS routinely throughout the night in addition to her NIV. She felt that her breathing had improved. Her PFTs three months postoperatively were FEV1 1.53 (53\% predicted), FVC 1.75 (48\% predicted), and MIP-49 (61\% predicted).

\section{Case 2}

A forty-eight year old, wheelchair-bound female diagnosed with ALS 18 months prior to presentation was referred to general surgery for placement of a DPS as treatment for respiratory insufficiency. She began using NIV for 6 months prior continuously at night and intermittently during the daytime. She also noted worsening dys-

Table 1. Induction of anesthesia.

Propofol 2 mg/kg

Remifentanil $50 \mathrm{mcg}$

Table 2. Maintenance of anesthesia.

Remifentanil infusion $0.1-0.2 \mathrm{mcg} / \mathrm{kg} / \mathrm{min}$

Sevoflourane 0.5 - 1 MAC 
phagia and fatigue with meals. Her preoperative FVC was 2.0 (79\% predicted), FEV1 2.58 (89\% predicted) and MIP-40 (48\% predicted).

She was taken to the OR and given 50mcg bolus of remifentanil and propofol (2 mg/kg). The patient was subsequently intubated without paralysis.

Anesthesia was maintained with sevoflurane and a remifentanil infusion. At the end of the surgery, the patient was allowed to breathe spontaneously and extubated in the operating room when she met criteria.

Over the next few months following the surgery, the patient began using the DPS system continuously during the day. This eliminated the need for daytime NIV. She continues to use NIV at nighttime. Her PFT two months postoperatively were FVC 1.67 (45\% predicted) FEV1 1.57 (54\% predicted), the patient was intolerant of respiratory pressure maneuvers, so her MIP could not be measured.

\section{Case 3}

A sixty-nine year old female diagnosed with ALS two years prior was referred to general surgery for placement of DPS. She is primarily wheelchair bound but able to walk short distances with assistance. She use nightly NIV for five months prior to presentation for evaluation of the DPS due to low MIP. Her preoperative PFTs were FVC 2.2 (70\% predicted), FEV1 1.98 (84\% predicted), MIP-32 (61\% predicted).

Induction of anesthesia and intubation were achieved with remifentanil and propofol boluses. Again, no neuromuscular blocking drugs were used. Maintenance of anesthesia was maintained with sevoflurane and remifentanil infusion. At the conclusion of the case sevoflurane and remifentanil were discontinued and the patient was allowed to breathe spontaneously. She did not meet extubation criteria in the operating room and was transported to the PACU with the ETT left in place. She was extubated in PACU approximately 45 minutes after surgery and was started on NIV at her home settings.

Postoperatively, the patient began using the DPS continuously and noticed a marked improvement in breathing. She no longer felt exertional dyspnea. She continued to use NIV at night. PFTs three months after surgery were as follows FVC 2.2 (70\% predicted), FEV1 1.72 (72\% predicted), and MIP 32 (48\% predicted).

\section{Discussion}

In the three cases described, the same anesthetic technique was employed. Two patients were extubated at the conclusion of surgery in the operating room. One patient was extubated in the PACU. The DPS was not used in the immediate postoperative period. All three patients continued to use NIV during the nighttime hours. The one patient who was using NIV during the day was able to discontinue it with the use of the DPS. Given the progressive nature of ALS, it is difficult to define how the DPS helped slow the rate of decline in respiratory function. Although there is a large degree of variability, it is estimated that FVC decreases by $3 \%$ - 5\% per month [2]. In the first case, the FVC declined by $14 \%$ in three months. In case two, the FVC declined by $34 \%$ in two months. And the third case remained unchanged.

Onders and colleagues described their experience with 51 patients undergoing DPS placement over the course of three years. Their technique included generous local anesthestic infiltrated at port sites at the start of the surgery. Propofol and remifentanil was used for induction and a remifentanil infusion and sevoflurane as maintenance. Morphine or dilaudid was also given as a long acting narcotic. Pulmonary compliance was measured in four patients at the end of the case. There was a $19 \%$ increase in respiratory compliance when the DPS was used. They postulated this change was primarily in the posterior lobes and decreased atelectasis. In their series there was a $0 \%$ mortality rate compared to $25 \%$ that has been reported in placing gastrostomy tubes in patients with ALS [4].

Schmiesing and colleagues published a case series of three patients. They also used a remifentanil infusion that was initiated at induction but did not give a remifentanil bolus. They induced with propfol, ventilated with $3 \%$ sevoflurance and topicalized using a lidocaine LTA in order to optimize intubating conditions. Anesthetic depth was maintained with the remifentanil infusion, $70 \%$ nitrous oxide, and end tidal sevoflurane of $1.5 \%$. All three patients were transferred to PACU intubated. The DPS was not used in the immediate postoperative period [6].

\section{Conclusions}

Anesthesia for the placement of the diaphragmatic pacemakers presents unique challenges. In patients with se- 
verely compromised respiratory function such as many ALS patients, extubation and general anesthesia may pose a significant additional risk. Since the surgeon needs to test muscle contraction of the diaphragm for placement of electrodes, neuromuscular blocking drugs cannot be administered. Additionally, in patients with ALS, succinylcholine should be avoided due to the risk of hyperkalemia.

Remifentanil, an ultra-short-acting opioid, provides a superb adjunct to sevoflurane for the facilitation of general anesthesia. Remifentanil depresses the respiratory drive sufficiently to facilitate mapping of the diaphragm during surgery. At the discontinuation of the infusion, the rapid offset of remifentanil allows the patient to resume normal respiration rapidly.

Given their preoperative pulmonary function, patients with ALS are high risk for surgery. They can be difficult to wean from the ventilator and are at risk for hypoventilation, aspiration and other postoperative pulmonary complications.

NIV may be necessary in the immediate post-operative period. Although not used in our three cases, the anesthesiologist can work with the programmer to use the stimulator in the postoperative period to facilitate extubation and improve pulmonary function in the immediate post-operative period.

\title{
References
}

[1] Bach, J.R. (1995) Amyotrophic Lateral Sclerosis: Predictors for Prolongation by Noninvasive Respiratory Aids. Archives of Physical Medicine and Rehabilitation, 76, 828-832. http://dx.doi.org/10.1016/S0003-9993(95)80547-8

[2] Onders, R.P., Elmo, M., Khansarinia, S., Bowman, B., Yee, J., Road, J., et al. (2009) Complete Worldwide Operative Experience in Laparoscopic Diaphragm Pacing: Results and Differences in Spinal Cord Injured Patients and Amyotrophic Lateral Sclerosis Patients. Surgical Endoscopy, 23, 1433-1440. http://dx.doi.org/10.1007/s00464-008-0223-3

[3] Forbes, R.B., Colville, S. and Swingler, R.J. (2004) Frequency, Timing and Outcome of Gastrostomy Tubes for Amyotrophic Lateral Sclerosis/Motor Neurone Disease. Journal of Neurology, 251, 813-817. http://dx.doi.org/10.1007/s00415-004-0429-9

[4] Onders, R.P. (2009) Amyotrophic Lateral Sclerosis: The Midwestern Surgical Experience with the Diaphragm Pacing Stimulation System Shows That General Anesthesia Can Be Safely Performed. The American Journal of Surgery, 197, 386-390. http://dx.doi.org/10.1016/j.amjsurg.2008.11.008

[5] Mahajan, K.R. (2012) Diaphragm Pacing and Noninvasive Respiratory Management of Amyotrophic Lateral Sclerosis/Motor Neuron Disease. Muscle and Nerve, 46, 851-855.

[6] Schmiesing, C.A., et al. (2010) Laparoscopic Diaphragmatic Pacer Placement-A Potential New Treatment for ALS Patients: A Brief Description of the Device and Anesthetic Issues. Journal of Clinical Anesthesia, 22, 549-552.

http://dx.doi.org/10.1016/j.jclinane.2009.09.010

\section{Abbreviations}

\author{
ALS: Amyotrophic Lateral Sclerosis \\ DPS: Diaphragm Pacing System \\ FVC: Forced Vital Capacity \\ FEV1: Forced Expiratory Volume in One Second \\ MIP: Maximal Inspiratory Pressure \\ PFT: Pulmonary Function Test \\ NIV: Non-Invasive Ventilation \\ OR: Operating Room \\ PACU: Post Anesthesia Care Unit \\ ETT: Endotracheal Tube \\ LTA: Laryngeal Tracheal Anesthesia
}


Scientific Research Publishing (SCIRP) is one of the largest Open Access journal publishers. It is currently publishing more than 200 open access, online, peer-reviewed journals covering a wide range of academic disciplines. SCIRP serves the worldwide academic communities and contributes to the progress and application of science with its publication.

Other selected journals from SCIRP are listed as below. Submit your manuscript to us via either submit@scirp.org or Online Submission Portal.
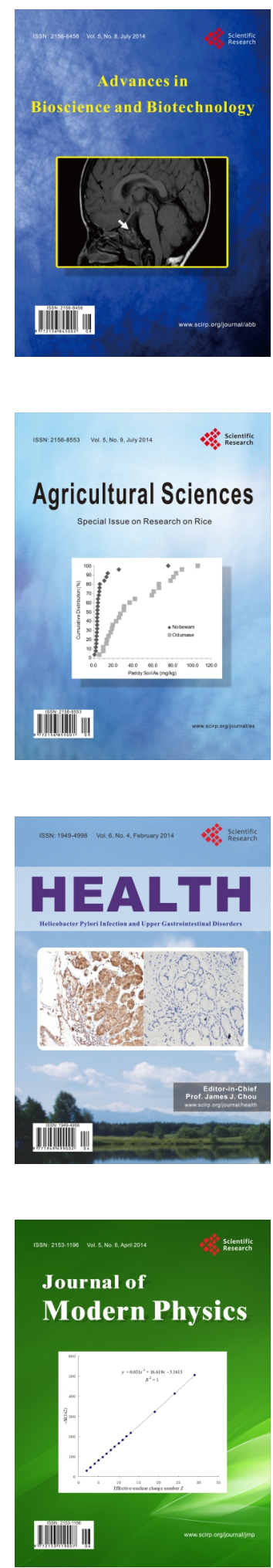
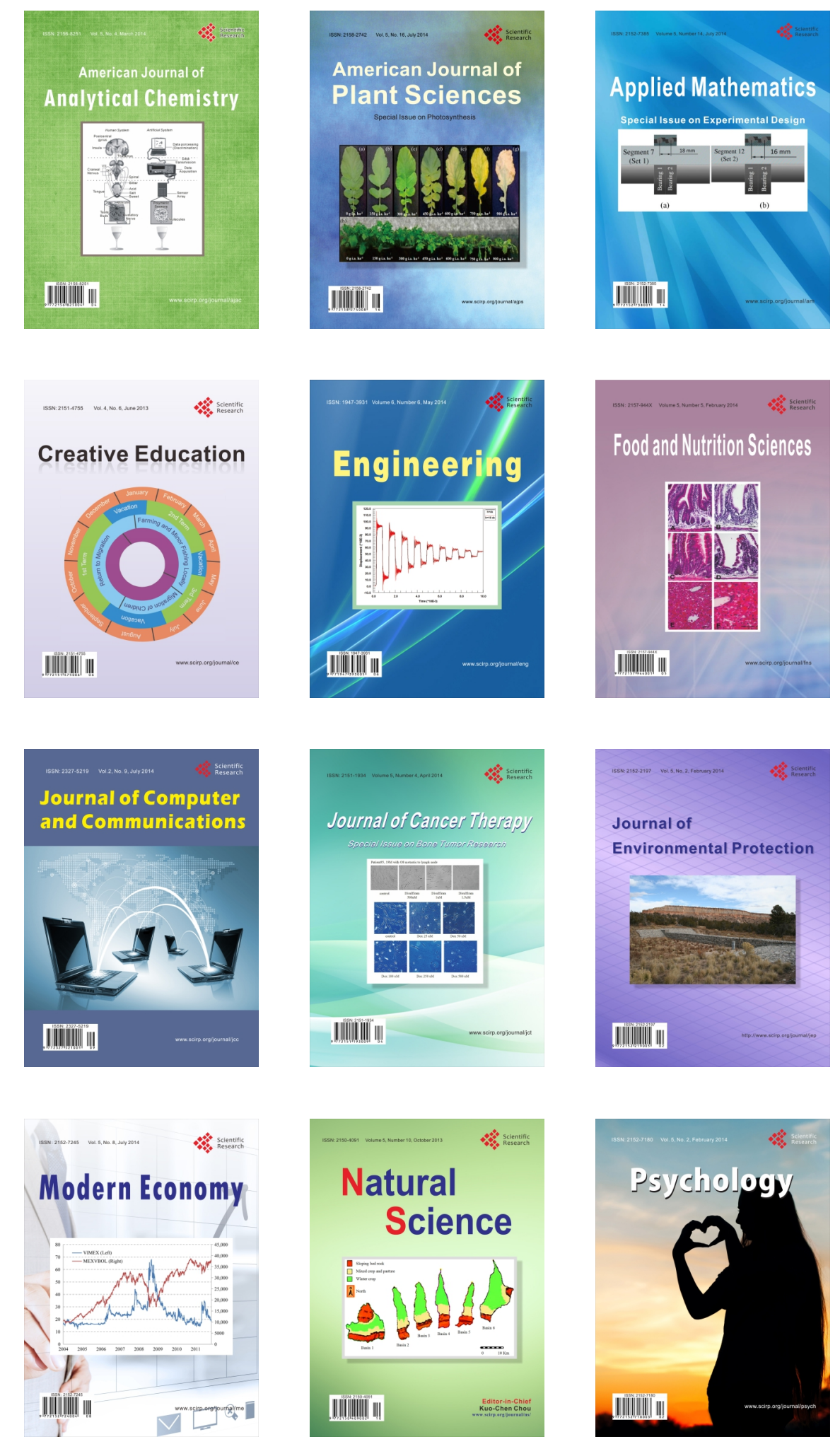